\title{
Distribución de genes codificadores de $\beta$-lactamasas de espectro extendido en aislamientos de Klebsiella pneumoniae de hospitales de Bogotá, D.C., Colombia
}

\author{
Ingrid Yamile Pulido, José Ramón Mantilla, Emilia María Valenzuela,
} María Teresa Reguero, Elsa Beatriz Gonzalez

\author{
Laboratorio de Epidemiología Molecular, Instituto de Biotecnología, Universidad Nacional de Colombia,
} Bogotá, D.C., Colombia

Introducción. Las beta-lactamasas de espectro extendido son las enzimas de Enterobacteriaceae de mayor distribución en Latinoamérica. No obstante, en Colombia existe poca información sobre la identidad de los genes codificadores de estas enzimas en Klebsiella pneumoniae.

Objetivo. Identificar genes bla presentes en $K$. pneumoniae procedentes de hospitales de Bogotá, D.C., Colombia.

Materiales y métodos. Se recolectaron 177 aislamientos productores de beta-lactamasas de espectro extendido de 10 hospitales de Bogotá, entre 2003 y 2005. Se determinó su sensibilidad antibiótica por difusión en disco y el número de beta-lactamasas presentes por isoelectroenfoque. Se identificaron los genes bla $a_{\mathrm{CTX}-\mathrm{M}}$, bla $\mathrm{SHV}_{\mathrm{SH}}$ y bla $\mathrm{TEM}_{\mathrm{TE}}$, mediante PCR y posterior secuenciación.

Resultados. Además de la resistencia a las cefalosporinas de tercera generación, el 44,7\% y el $49,7 \%$ fueron resistentes a la gentamicina y al trimetoprim-sulfametoxazol, respectivamente. Se observaron porcentaje de resistencia más bajos con otros antibióticos. En promedio, se detectaron por aislamiento tres beta-lactamasas, y los genes bla CTX-M-12 $_{2}(56 \%)$ y bla SHV-12 $_{2}(33,3 \%)$ fueron los más prevalentes. Además, se identificaron bla ${ }_{\mathrm{SHV}-5}(11,8 \%)$, bla $\mathrm{CTX}-\mathrm{M}-1_{1}(4 \%)$, bla SHV-27 $(2,8 \%)$, bla $\mathrm{SHV}-2(2,8 \%)$, bla $_{\text {СТX-м-2 }}(1,7 \%)$ y bla CTX-M-15 $(0,6 \%)$. Además, en nuestros aislamientos se identificaron tres genes codificadores de beta-lactamasas de espectro ampliado.

Conclusión. Se identificaron once genes bla, de los cuales, ocho eran codificadores de beta-lactamasas de espectro extendido. La diversidad encontrada de los genes bla sugiere la continua exposición de $K$. pneumoniae a fuertes presiones antibióticas, como las observadas en nuestros hospitales.

Palabras clave: Klebsiella pneumoniae, beta-lactamasas, farmacorresistencia bacteriana, análisis de secuencia de ADN, epidemiología molecular, Colombia.

Distribution of extended spectrum $\beta$-lactamases-codifying genes in Klebsiella pneumoniae isolates from hospitals of Bogota, D.C., Colombia

Introduction. Extended spectrum $\beta$-lactamases (ESBL) are the most widely distributed enzymes in Enterobacteriaceae of Latin America and are key enzymes in resistance to antibiotics in common use. However, in Colombia, little information is available concerning the identity of genes coding for these enzymes in Klebsiella pneumoniae.

Objective. The bla genes were identified in K. pneumoniae isolated from hospitals in Bogotá D.C., Colombia.

Materials and methods. One hundred seventy-seven isolates of ESBL producers were collected from 10 hospitals in Bogota between 2003 and 2005. Antibiotic susceptibility was determined by disk diffusion, and the number of $\beta$-lactamases in each isolate was assessed by isoelectric focusing. bla $a_{\text {CTX-M, }}$ $b / a_{\mathrm{SHV}}$ and $b / a_{\mathrm{TEM}}$ were identified by PCR and subsequent sequencing.

Results. Besides, the resitenance to third generation cephalosporins, $44.7 \%$ and $49.7 \%$ were resistant to amikacyn and thrimetoprim-sulaphametoxazole respectively. Lower resistance rates to other antibiotics were observed as well. An average of three $\beta$-lactamases were detected by isoelectric focusing, and the genes $b / a_{\mathrm{CTX}-\mathrm{M}-12}(56.0 \%)$ and $b / a_{\mathrm{SHV}-12}(33.3 \%)$ were the most prevalent. bla $\mathrm{SHV}_{\mathrm{SH}}(11.8 \%)$, bla $a_{\mathrm{CTX}-\mathrm{M}-1}$ $(4.0 \%)$, bla $\mathrm{SHV}-27(2.8 \%)$, bla $a_{\mathrm{SHV}-2}(2.8 \%)$, bla $\mathrm{CTX}_{\mathrm{CT}-2}(1.7 \%)$ and bla $\mathrm{CTX}-\mathrm{M}-15(0.6 \%)$ were present in smaller percentages. In addition, three genes were identified that coded for narrow spectrum $\beta$-lactamases.

Conclusion. Eleven bla genes were identified, eight of which were ESBL-coding. The diversity of the bla genes suggested a continuing exposure of $K$. pneumoniae to strong antibiotic pressures in Bogota hospitals.

Key words: Klebsiella pneumoniae; beta-lactamases; drug resistance, bacterial; sequence analysis, DNA; molecular epidemiology; Colombia. 
El uso masivo e indiscriminado de antibióticos en los hospitales ha conducido a la selección y permanencia de microorganismos multirresistentes de difícil erradicación. La producción de beta-lactamasas es el principal mecanismo de resistencia exhibido por miembros de la familia Enterobacteriaceaey, particularmente, en Klebsiella pneumoniae, uno de los patógenos hospitalarios más comúnmente asociados con la producción de estas enzimas $(1,2)$.

La localización de los genes codificadores de betalactamasas de espectro extendido y de otros genes de resistencia a antibióticos no beta-lactámicos en plásmidos transferibles, ha permitido su diseminación entre diferentes especies de enterobacterias. En la actualidad, es común encontrar microorganismos resistentes a varios grupos de antibióticos que incluyen no sólo beta-lactámicos, sino también aminoglucósidos, quinolonas y sulfonamidas (3-5). Con este panorama, las opciones terapéuticas para el tratamiento de las infecciones causadas por estos microorganismos son cada vez más limitadas (6).

En Latinoamérica, el fenotipo de beta-lactamasas de espectro extendido en enterobacterias se encuentra con una gran frecuencia, en comparación con otras áreas geográficas (7). En Colombia, desde 1997, los estudios de vigilancia de la resistencia a beta-lactámicos han reportado la existencia de $K$. pneumoniae resistente a cefalosporinas de tercera generación por producción de beta-lactamasas de espectro extendido $(8,9)$. Otros estudios más recientes en el país han descrito, en esta especie, la presencia de los genes codificadores de betalactamasas de espectro extendido derivadas de las familias SHV y CTX-M (10-12). Sin embargo, existe poca información significativa sobre la identidad y distribución de estos genes en aislamientos de $K$. pneumoniae obtenidos de pacientes internados en centros hospitalarios del país.

La contribución de este estudio fue establecer la diversidad de genes bla codificadores de betalactamasas de espectro extendido en aislamientos de K. pneumoniae obtenidos de 2003 a 2005,

\footnotetext{
Correspondencia:

Elsa Beatriz González, Laboratorio de Epidemiología Molecular, Instituto de Biotecnología, Universidad Nacional de Colombia, Ciudad Universitaria, Bogotá, D.C., Colombia.

Teléfono: (571) 316 5000, extensión: 16965.

ebgonzalezm@unal.edu.co

Recibido: 18/08/09; aceptado:07/09/10
}

de pacientes de 10 hospitales de tercer nivel de complejidad de Bogotá. Todos los aislamientos fueron productores de beta-lactamasas de espectro extendido y causantes en su mayoría de infecciones intrahospitalarias.

\section{Materiales y métodos}

Se analizaron 177 aislamientos no clonales recolectados durante los años 2003, 2004 y 2005, de $K$. pneumoniae de 10 hospitales de Bogotá. Todos los aislamientos fueron nuevamente identificados mediante el sistema API ${ }^{\circledR}$ (Biomerieux, Hazelwood, MO) y se les hizo la prueba confirmatoria de betalactamasas de espectro extendido recomendada por el Clinical and Laboratory Standards Institute (CLSI) (13). Las cepas Escherichia coli ATCC 25922 y K. pneumoniae ATCC 700603 fueron utilizadas como referencia en los ensayos de sensibilidad antibiótica.

\section{Ensayos de sensibilidad}

Lasensibilidad a diferentes antibióticos se determinó por el método de difusión en agar, de acuerdo con los parámetros establecidos por el CLSI (13) en todos los aislamientos evaluados. Se utilizaron discos de: amikacina $(30 \mu \mathrm{g})$, aztreonam $(30 \mu \mathrm{g})$, cefotaxima $(30 \mu \mathrm{g})$, cefepime $(30 \mu \mathrm{g})$, ceftazidima $(30 \mu \mathrm{g})$, ceftriaxona $(30 \mu \mathrm{g})$, ciprofloxacina (5 $\mu \mathrm{g})$, gentamicina $(10 \mu \mathrm{g})$, imipenem $(10 \mu \mathrm{g})$, piperacilina-tazobactam $(100 / 10 \mu \mathrm{g})$ y trimetoprimsulfametoxazol $(1,25 / 23,75 \mu \mathrm{g})$ (Oxoid(B).

\section{Determinación de puntos isoeléctricos}

El ensayo de isoelectroenfoque analítico se hizo con extractos celulares obtenidos por sonicación de $30 \mathrm{ml}$ de cultivos de 18 horas en caldo LB. Se utilizó el sistema mini-IEF cell@ modelo 111(BioRad), con geles de poliacrilamida (25\% T, 3\% C) y bajo las recomendaciones del fabricante. Como puntos isoeléctricos de referencia se incluyeron extractos celulares de cepas bacterianas que expresaban diferentes beta-lactamasas (TEM-1: 5,4; TEM-10: 5,6; SHV-7: 7,6; SHV-5: 8,2 y CTX-M12: 8,9) у el marcador visible estándar-IEF® (BioRad). La actividad de las beta-lactamasas enfocadas se reveló por el método iodométrico con ampicilina $(500 \mu \mathrm{g} / \mathrm{ml})$ como sustrato (14).

\section{Detección de genes bla}

Los genes codificadores de enzimas de betalactamasas de espectro extendido de los tipos SHV, TEM y CTX-M (grupos 1, 2, 8 y 9) se detectaron en los aislamientos de estudio por la reacción en cadena de la polimerasa (PCR). Para ello, se utilizaron los 
iniciadores OS-5 y OS-6 (15) para la detección de genes bla $a_{\mathrm{SHV}}$ y los iniciadores $\mathrm{J}$ y $\mathrm{E}$ (16) para los genes $b a_{\mathrm{TEM}}$. En la detección de genes $b l a_{\mathrm{CTX}-\mathrm{M}}$, se utilizaron los iniciadores CTX-MA y CTX-MB para el grupo 1 (17), los iniciadores TOHO1A y TOHO1B para el grupo 2 (18) y los C1 y C2 para el grupo 9 (19). Para la detección de los genes bla ${ }_{\text {CTX-M }}$ del grupo 8 se utilizaron los iniciadores CTX-M-8-F y CTX-M-8-R (20). Se realizaron mezclas de reacción con concentraciones de 0,2 mM de dNTPs, $1,5 \mathrm{mM}$ de $\mathrm{MgCl}_{2}, 1 \mu \mathrm{M}$ cada iniciador y $0,04 \mathrm{U} / \mu$ l unidades de TaqDNA polimerasa (Invitrogen®).

EI ADN molde se obtuvo de colonias bacterianas frescas sometidas a calentamiento hasta ebullición durante 10 minutos. La amplificación se hizo bajo condiciones estandarizadas $\left(95^{\circ} \mathrm{C}\right.$ por 5 minutos, 30 ciclos de $95{ }^{\circ} \mathrm{C}$ por un minuto, $57^{\circ} \mathrm{C}$ por un minuto, $72{ }^{\circ} \mathrm{C}$ por un minuto y una extensión final a $72{ }^{\circ} \mathrm{C}$ por 5 minutos) (termociclador iCycler ${ }^{\circledR}$ de Bio-Rad). Los amplificados se analizaron en geles de agarosa al $1 \%$, teñidos con bromuro de etidio $(0,5 \mu \mathrm{g} / \mathrm{ml})$ en solución tampón TBE 0,5X. Se utilizó como referencia de tamaños, el marcador DNA $100 \mathrm{pb}$ ladder (Invitrogen®).

\section{Secuenciación y análisis computacional de las secuencias}

Los productos de la amplificación obtenidos se purificaron y secuenciaron en ambas cadenas, utilizando los iniciadores antes descritos y con equipos de secuenciación automatizados (servicio de secuenciación Macrogen, Corea ABI3730XL de Applied Biosystems). Las secuencias obtenidas se compararon con la ayuda de la herramienta BLAST, con las secuencias de ADN almacenadas en bases públicas de datos (disponible en: http:// www.ncbi.nlm.nih.gov/).

\section{Resultados}

\section{Ensayos de sensibilidad}

Los antibiogramas mostraron que 154 de los 177 aislamientos (87\%) eran resistentes a cefotaxima y a ceftriaxona. Ciento nueve aislamientos $(61,5$ $\%)$ presentó resistencia a ceftazidima y 66 de los 177 aislamientos fueron resistentes a aztreonam $(37,2 \%)$. Se observó resistencia al cefepime en $28,8 \%$ de los aislamientos. Los resultados obtenidos para la combinación antibiótico-inhibidor (piperacilina/tazobactam) indicaron un 25,3\% de aislamientos resistentes. Para otros antibióticos, se encontraron $84(47,4 \%)$ aislamientos resistentes a amikacina y $88(49,7 \%)$ resistentes a trimetoprim/ sulfametoxazol. Se detectó resistencia simultánea a ciprofloxacina $(11,3 \%)$ y gentamicina $(22 \%)$ se detectó en los aislamientos estudiados. Todos los aislamientos fueron sensibles a imipenem.

\section{Puntos isoeléctricos}

Se detectaron puntos isoeléctricos de 5,4, 6,8, $7,0,7,6,8,0,8,2$ y mayor de 8,7. En promedio, se encontraron tres puntos isoeléctricos para cada aislamiento en diferentes combinaciones. El perfil dominante presentó las beta-lactamasas con puntos isoeléctricos de 5,4, 7,6 y mayor de 8,7. El punto isoeléctrico que se detectó con mayor frecuencia fue 5,4 en 128 aislamientos, seguido por el mayor de 8,7 en 107; el punto 7,6 se detectó en 85 aislamientos y el punto 8,2 en 79. En menor proporción, se detectaron los puntos 7,0, 6,8 y 8,0 en 14,5 y 3 aislamientos, respectivamente (cuadro 1).

\section{Detección de genes bla}

Los resultados de amplificación para los genes bla $a_{\text {CTX-M }}$ fueron positivos para los genes del grupo 1 , y se obtuvieron amplificados en 107 de los 177 aislamientos y para el grupo 2, en 3 aislamientos de los totales del estudio. En 170 de los 177 aislamientos totales (96\%) se detectaron los genes $b l a_{\mathrm{SHV}}$ y en 128 de los $177(75,7 \%)$, del tipo bla $_{\mathrm{TEM}}$. Se hallaron combinaciones con los tres tipos de genes en 92 (52\%) de los 177 aislamientos, con bla $_{\text {CTX-M }}$ y bla $a_{\text {TEM }}$ en 7 aislamientos (4\%) y con los genes $b / a_{\mathrm{CTX}-\mathrm{M}}$ y bla $a_{\mathrm{SHV}}$ en $11(5,6 \%)$ de ellos. En $29(14,7 \%)$ aislamientos se detectaron los genes $b l a_{\mathrm{SHV}}$ y $b l a_{\mathrm{TEM}}$, y en 38 de los $177(21,5 \%)$ se detectaron únicamente los genes bla ${ }_{\mathrm{SHV}}$ (cuadro 1).

\section{Secuenciación y análisis computacional de las secuencias}

El análisis de las secuencias de los productos de amplificación obtenidos de aislamientos seleccionados para los genes bla $_{\text {CTX-M }}$, reveló $100 \%$ de similitud en ambas cadenas con la secuencias codificadoras de las enzimas CTX-M-12 (100/177; 56,5 \%), CTX-M-1(7/177; $4 \%$ ), CTX-M-2( 3/177; $1,7 \%)$ y CTX-M15 (1/177; 0,5 \%). De igual manera, las secuencias obtenidas a partir de los amplificados de genes $b a_{\mathrm{SHV}}$ presentaron $100 \%$ de similitud con las secuencias codificadoras de las enzimas SHV-12 (59/177; 33,3 \%), SHV-5 (21/177; $11,8 \%)$, SHV-2 (5/177; 2,8 \%), SHV-27(5/177; 2,8 $\%)$, SHV-11(3/177; $1,7 \%)$ y SHV-1 (77/177; 43,5 $\%)$; las secuencias amplificadas del gen $b a_{\text {TEM }}$ en todos los casos correspondieron con aquellas de los genes codificadores de las enzimas TEM-1 y sus variantes (TEM-1B y TEM-1E). 


\section{Discusión}

Klebsiella pneumoniae es uno de los patógenos hospitalarios más asociados no sólo con infecciones respiratorias, sino tambien, con infecciones de las vías urinarias, septicemias e infecciones de heridas, entre otras (21-24). La acentuada resistencia a cefalosporinas de espectro extendido encontrada en este microorganismo, limitan cada vez más el uso de estos antibióticos en infecciones causadas por estos gérmenes.

Los resultados observados para los puntos isoeléctricos y los genes codificadores detectados mediante PCR de las enzimas beta-lactamasas, son concordantes entre sí y con los resultados de secuenciación. Así, en los aislamientos en los cuales se detectaron los genes bla ${ }_{\mathrm{CTX}-\mathrm{M}}$, se detectó la producción de enzimas con puntos isoeléctricos de 8,2 y mayor de 8,7 propios de las beta-lactamasas de espectro extendido CTX-M-2, CTX-M-1, CTXM-12 y CTX-M-15. La enzima CTX-M-12, fue la predominante en los aislamientos estudiados de $K$. pneumoniae.
Esta beta-lactamasa se reportó por primera vez en Kenia, en el 2003, y en Colombia, en el $2004(25,26)$. Otras enzimas de esta familia han sido reportadas en aislamientos de enterobacterias previamente (10). En menor proporción, se encontraron los genes de resistencia para las beta-lactamasas de espectro extendido SHV-12, SHV-5, SHV-2 y SHV- 27, esta última reportada por primera vez en el país en esta investigación y que, a diferencia de las otras SHV detectadas, presenta una mayor actividad hidrolítica sobre la cefotaxima (27). No se detectó un gen que pudiera ser asociado con el punto isoeléctrico de 7,0 .

La diversidad de genes encontrada en este estudio se asemeja a las observadas en países asiáticos y contrasta con las presentes en Estados Unidos y Europa, que reflejan, posiblemente, ambientes con presiones antibióticas diferentes (19).

Al igual que otros reportados, este estudio encontró que miembros de la familia Enterobacteriaceae, y específicamente en $K$. pneumoniae, presentan la capacidad de acumular varias enzimas hidrolíticas

Cuadro 1. Combinaciones de genes bla identificados y puntos isoeléctricos detectados en aislamientos de Klebsiella pneumoniae productores de beta-lactamasas de espectro extendido.

\begin{tabular}{|c|c|c|c|c|c|}
\hline $\begin{array}{l}\text { Combinaciones de genes } \\
\text { bla detectadas } \\
\text { por PCR } \\
\text { bla }_{\mathrm{SHV}} \text { bla }{ }_{\mathrm{CTX}-\mathrm{M}} \text { y bla } \\
\mathrm{TEM}\end{array}$ & $\begin{array}{c}\begin{array}{c}\text { Combinación de genes } \\
\text { bla identificadas } \\
\text { por secuenciación }\end{array} \\
\text { SHV-1, CTX-M-12, TEM-1 }\end{array}$ & $\begin{array}{c}\begin{array}{c}\text { Número de } \\
\text { aislamientos }\end{array} \\
63\end{array}$ & \multicolumn{3}{|c|}{$\begin{array}{c}\text { Puntos isoeléctricos } \\
\text { detectados }\end{array}$} \\
\hline bla $_{\mathrm{SHV}}$, bla ${ }_{\mathrm{CTX}-\mathrm{M}}$ y bla & $\begin{array}{l}\text { SHV-1, CTX-M-12, TEM-1 } \\
\text { SHV-1, CTX-M-12, TEM-1E } \\
\text { SHV-1, CTX-M2, TEM-1 } \\
\text { SHV-1, CTX-M-1, TEM-1 } \\
\text { SHV-2, CTX-M-12, TEM-1 } \\
\text { SHV-5, CTX-M-12, TEM-1 } \\
\text { SHV-5, CTX-M-12, TEM-1B } \\
\text { SHV-11, CTX-M-12, TEM -1 } \\
\text { SHV-11, CTX-M-2, TEM -1 } \\
\text { SHV-11, CTX-M-15, TEM -1 } \\
\text { SHV-12, CTX-M12, TEM-1 } \\
\text { SHV-27, CTX-M-12, TEM-1 } \\
\text { Subtotal }\end{array}$ & $\begin{array}{r}63 \\
1 \\
2 \\
1 \\
4 \\
3 \\
2 \\
1 \\
1 \\
1 \\
9 \\
4 \\
92\end{array}$ & $\begin{array}{l}7,6 \\
7,6 \\
7,6 \\
7,6 \\
7,6 \\
8,2 \\
8,2 \\
7,6 \\
7,6 \\
7,6 \\
8,2 \\
6,8\end{array}$ & $\begin{array}{c}>8,7 \\
>8,7 \\
8 \\
>8,7 \\
>8,7 \\
>8,7 \\
>8,7 \\
>8,7 \\
8 \\
>8,7 \\
>8,7 \\
>8,7\end{array}$ & $\begin{array}{l}5,4 \\
5,4 \\
5,4 \\
5,4 \\
5,4 \\
5,4 \\
5,4 \\
5,4 \\
5,4 \\
5,4 \\
5,4 \\
5,4\end{array}$ \\
\hline bla $_{\mathrm{SHV}} \mathrm{y}$ bla ${ }_{\mathrm{CTX}-\mathrm{M}}$ & $\begin{array}{l}\text { SHV-1, CTX-M-12 } \\
\text { SHV-1, CTX-M-1 } \\
\text { SHV-27, CTX-M-1 } \\
\text { Subtotal }\end{array}$ & $\begin{array}{r}5 \\
5 \\
1 \\
11\end{array}$ & $\begin{array}{l}7,6 \\
7,6 \\
6,8\end{array}$ & $\begin{array}{l}>8,7 \\
>8,7 \\
>8,7\end{array}$ & \\
\hline bla $_{\mathrm{SHV}}$ y bla & $\begin{array}{l}\text { SHV-2, TEM-1 } \\
\text { SHV-12, TEM-1 } \\
\text { SHV-5, TEM-1 } \\
\text { Subtotal }\end{array}$ & $\begin{array}{r}1 \\
22 \\
6 \\
29\end{array}$ & $\begin{array}{l}7,6 \\
8,2 \\
8,2\end{array}$ & & $\begin{array}{l}5,4 \\
5,4 \\
5,4\end{array}$ \\
\hline$b_{\text {CTX-M }}$ y bla ${ }_{\mathrm{TEM}}$ & $\begin{array}{l}\text { CTX-M-12, TEM-1 } \\
\text { Subtotal }\end{array}$ & $\begin{array}{l}7 \\
7\end{array}$ & & $>8,7$ & 5,4 \\
\hline \multirow[t]{3}{*}{$b_{\text {sHv }}$} & $\begin{array}{l}\text { SHV-12 } \\
\text { SHV-5 }\end{array}$ & $\begin{array}{l}28 \\
10\end{array}$ & $\begin{array}{l}8,2 \\
8,2\end{array}$ & & \\
\hline & Subtotal & 38 & & & \\
\hline & Total de aislamientos & 177 & & & \\
\hline
\end{tabular}


de beta-lactámicos como estrategia adicional de resistencia, lo que les permite cubrir un rango amplio de antibióticos, desde las penicilinas y cefalosporinas de primera y segunda generación (sustrato de las beta-lactamasas de espectro ampliado), hasta las cefalosporinas de tercera y cuarta generación y monobactámicos (sustrato de las beta-lactamasas de espectro extendido) $(28,29)$. Así, los diferentes genes encontrados podrían explican la gran frecuencia de microorganismos resistentes a las cefalosporinas evaluadas. A lo anterior se le suman los porcentajes de resistencia a ciprofloxacina, gentamicina, amikacina y trimetoprimsulfametoxazol que evidencian el potencial de las bacterias estudiadas para adquirir y expresar resistencia hacia otros grupos de antibióticos y la cada vez más limitada disposición de antibióticos eficaces para tratar las infecciones causadas por estos microorganismos (29).

La frecuencia con la que se detectó la enzima TEM-1 en los aislamientos, corresponde con lo ya señalado para otros miembros de la familia Enterobacteriaceae (30) y sugiere, tal como se ha señalado previamente, la facilidad de estos microorganismos para adquirir enzimas con actividad de espectro extendido derivadas de la SHV-1, más que la presentación de mutaciones que originen variantes derivadas de la TEM-1 con actividad sobre las cefalosporinas de tercera generación (31).

Este estudio estableció la identidad y distribución de los genes codificadores de beta-lactamasas presentes en aislamientos de K. pneumoniae de 10 hospitales de Bogotá. Dichas bacterias presentaron diferentes combinaciones de enzimas que les confieren espectros diversos de hidrólisis hacia los diferentes antibióticos disponibles. Las resistencias observadas a otros antibióticos no beta-lactámicos enfatizan la importancia en las medidas de vigilancia y seguimiento que deben implementarse en los hospitales y el establecimiento de programas de control de antibióticos, que permitan frenar la diseminación de estos microorganismos multirresistentes.

\section{Agradecimientos}

Los autores agradecen la colaboración a los integrantes del Laboratorio de Microbiología de la Universidad de Buenos Aires, por brindar capacitación en técnicas moleculares, a la Secretaría Distrital de Salud y a los laboratorios de bacteriología de los hospitales participantes y al Departamento Administrativo de Ciencia, Tecnología e Innovación,
Colciencias, y la Dirección de Investigación de la Universidad Nacional de Colombia sede Bogotá, entidades responsables de la totalidad de la financiación del estudio.

\section{Conflicto de intereses}

Los autores declaran que no existen relaciones económicas ni personales de índole alguna que pudieran influenciar su juicio respecto a los resultados de la presente investigación. Todos los autores tuvieron acceso irrestricto a los datos y no exisitió participación de agentes externos en el diseño del estudio, ni en la recopilación, análisis e interpretación de la información.

\section{Financiación}

Este trabajo fue financiado en su totalidad con fondos provenientes del Departamento Administrativo de Ciencia, Tecnología e Innovación, Colciencias (código 1101-04-16483 CT 414-2004) y la Dirección de Investigación de la Universidad Nacional de Colombia, sede Bogotá.

\section{Referencias}

1. Martínez JL, Baquero F. Interactions among strategies associated with bacterial infection: Pathogenicity, epidemicity and antibiotic resistance. Clin Microbiol Rev. 2002;4:647-79.

2. Bonnet R. Growing group of extended-spectrum $\beta$-lactamases: The CTX-M enzymes. Antimicrob Agents Chemother. 2004;48:1-14.

3. Pagani L, Dell'Amico E, Migliavacca R, D'Andrea MM, Giacobone E, Amicosante G, et al. Multiple CTX-M-type extended-spectrum $\beta$-lactamases in nosocomial isolates of Enterobacteriaceae from a hospital in Northern Italy. J Clin Microbiol. 2003;41:4264-9.

4. Van't veen A, van der Zee A, Nelson J, Speelberg B, Kluytmans JA, Buiting AG. Outbreak of infection with a multiresistant Klebsiella pneumoniae strain associated with contaminated roll boards in operating rooms. J Clin Microbiol. 2005;43:4961-7.

5. Ben-Hamouda T, Foulon T, Ben-Cheikn-Masmoudi A, Fendri C, Belhadj O, Ben Mahrez K. Molecular epidemiology of an outbreak of multiresistant Klebsiella pneumoniae in a Tunisian neonatal ward. J Med Microbiol. 2003;52:427-33

6. Podschun R, Ullmann U. Klebsiella spp. as nosocomial pathogens: Epidemiology, taxonomy, typing methods, and pathogenicity factors. Clin Microbiol Rev. 1998;11:589-603.

7. Winokur PL, Canton R, Casellas JM, Legakis N. Variations in the prevalence of strains expressing an extendedspectrum $\beta$-lactamase phenotype and characterization of isolates from Europe, the Americas, and the Western Pacific region. Clin Infect Dis. 2001;32(Suppl. 2):S94-103.

8. The Colombian Resistance Antimicrobial Group, Jones RN, Salazar JC, Pfaller MA, Doern GV. Multicenter evaluation of antimicrobial resistance to six broad-spectrum 
$\beta$-lactams in Colombia using the Etest method. Diagn Microbiol Infect Dis. 1997;29:265-72.

9. The Colombian Antimicrobial Resistance Study Group, Pfaller MA, Jones RN, Doern GV, Salazar JC. Multicenter evaluation of antimicrobial resistance to six broad-spectrum beta-lactams in Colombia: Comparison of data from 1997 and 1998 using the Etest method. Diagn Microbiol Infect Dis. 1999;35:235-41.

10. Valenzuela E, Mantilla J, Reguero M, González E, Pulido I, Llerena I, et al. Detection of CTX-M-1, CTX-M-15, and CTX-M-2 in clinical isolates of Enterobacteriaceae in Bogotá, Colombia. J Clin Microbiol. 2005;44:1919-20.

11. Espinal P, Mantilla J, Saavedra C, Leal A, Alpuche C, Valenzuela E. Epidemiología molecular de la infección nosocomial por Klebsiella pneumoniae productora de beta-lactamasas de espectro extendido. Biomédica. 2004;24:252-61.

12. Villegas M, Correa A, Pérez F, Miranda MC, Zuluaga T, Quinn JP, et al. Prevalence and characterization of extended-spectrum $\beta$-lactamases in Klebsiella pneumoniae and Escherichia coli isolates from Colombian hospitals. Diagn Microbiol Infect Dis. 2004;49:217-22.

13. Clinical and Laboratory Standards Institute. Performance standards for antimicrobial susceptibillity testing. Sixteenth Informational supplement. CLSI document M-100-S16 (ISBN 1-56238-588-7). Wayne: Clinical and Laboratory Standards Institute; 2006.

14. Jarlier V, Nicolas MH, Fournier G, Philippon A. Extended broad-spectrum $\beta$-lactamases conferring transferable resistance to newer $\beta$-lactams agents in Enterobacteriaceae: Hospital prevalence and susceptibility patterns. Rev Infect Dis. 1988;10:867-78.

15. Cao V, Lambert T, Quynh Nhu D, Loan HK, Hoang N, Arlet G, et al. Distribution of extended-spectrun $\beta$-lactamases in clinical isolates of Enterobacteriaceae in Vietnam. Antimicrob Agents Chemother. 2002;46:3739-43.

16. Neuwirth C, Madec S, Siebor E. TEM-89 $\beta$-lactamase produced by Proteus mirabilis clinical isolate: New complex mutant (CMT 3) with mutations in both TEM-59 (IRT-17) and TEM-3. Antimicrob Agents Chemother. 2001;45:3591-4.

17. Bonnet R, Dutour J, Sampaio M, Chanal C, Sirot D, Labia R, et al. Novel cefotaxime (CTX-M-16) with increase catalytic efficiency due to substitution Asp-240-Gli. Antimicrob Agents Chemother. 2001;45:2269-75.

18. Nagano N, Shibata N, Saitou Y, Nagano Y, Arakawa Y. Nosocomial outbreak infections by Proteus mirabilis that produces extended-spectrum CTX-M-2 type $\beta$-lactamase. J Clin Microbiol. 2003;41:5530-6.

19. Chanawong A, M'Zali FH, Heritage J, Xiong J, Hawkey PM. Three cefotaximases, CTX-M-9, CTX-M-13, and CTXM-14, among Enterobacteriaceae in the People's Republic of China. Antimicrob Agents Chemother. 2002;46:630-7.

20. Shibata N, Kurokawa H, Doi Y, Yagi T, Yamane K, Wachino $J$, et al. PCR classification of CTX-M-type $\beta$-lactamase genes identified in clinically isolated gram-negative bacilli in Japan. Antimicrob Agents Chemother. 2006;50:791-5.

21. Bouza E, San Juan R, Muñoz P, Voss A, Kluytmans J. A European perspective on nosocomial urinary tract infections II. Report on incidence, clinical characteristics and outcome (ESGNI-004study). Clin Microbiol Infect. 2001;7:532-42.

22. Kil K, Darouiche RO, Hull RA, Mansouri MD, Musher DM. Identification of a Klebsiella pneumoniae strain associated with nosocomial urinary tract infection. J Clin Microbiol. 1997;35:2370-4.

23. Suljagic V, Cobeljic M, Jankovic S, Mirovic V, MarkovicDenic L, Romic $\mathbf{P}$, et al. Nosocomial bloodstream infections in ICU and non-ICU patients. Am J Infect Control. 2005;33:333-40.

24. Biedenbach DJ, Moet GJ, Jones RN. Ocurrence and antimicrobial resistance pattern comparisons among bloodstream infection isolates from the SENTRY Antimicrobial Surveillance Program (1997- 2002). Diag Microbiol Infect Dis. 2004;50:59-69.

25. Kariuki S, Corkill J, Revathi G, Musoke R, Hart CA. Molecular characterization of a novel plasmid-encodedcefotaximase (CTX-M-12) found in clinical Klebsiella pneumoniae isolates from Kenya, Antimicrob Agents Chemother. 2001;45:2141-3

26. Villegas MV, Correa A, Pérez F, Zuluaga $T$, Radice $M$, Gutkind G, et al. CTX-M-12 $\beta$-lactamase in a Klebsiella pneumoniae isolate in Colombia. Antimicrob Agents Chemother. 2004;48:629-31.

27. Corkill JE, Cuevas LE, Gurgel RQ, Greensill J, Hart CA. SHV-27, a novel cefotaxime-hydrolyzing B-lactamase, identified in Klebsiella pneumoniae isolates from a Brazilian hospital. J Antimicrob Chemother. 2001;47:463-5.

28. Segatore B, Setacci D, Perilli M, Franchino L, Franceschini N, Agnifili A, et al. Antimicrobial susceptibility of clinical isolates of Enterobacteriaceae producing complex $\beta$-lactamase patterns including extended-spectrum enzymes. Int J Antimicrob Agents. 2004;23:480-6.

29. Xiong Z, Zhu D, Wang F, Zhang Y, Okamoto R, Inoue M. A Klebsiella pneumoniae producing three kinds of class A $\beta$-lactamases encoded by one single plasmid isolated from a patient in Huashan Hospital, Shanghai, China. Int J Antimicrob Agents. 2004;23:262-7.

30. Bradford PA. Extended-spectrum $\beta$-lactamases in the 21st century: Characterization, epidemiology and detection of this important resistance threat. Clin Microbiol Rev. 2001;14:933-51.

31. Siu LK, Lu P, Hsueh P, Lin FM, Chang S, Luh K, et al. Bacteremia due to extended-spectrum $\beta$-lactamaseproducing Escherichia coli and Klebsiella pneumoniae in a pediatric oncology ward: Clinical features and identification of different plasmids carrying both SHV-5 and TEM-1 genes. J Clin Microbiol. 1999;37:4020-7. 